\title{
Emission Line Region in a sample of 12 active galactic nuclei
}

\author{
L. Č. Popović ${ }^{1,2}$, E. Mediavilla ${ }^{3}$ E. Bon ${ }^{1}$ and D. Ilić ${ }^{4}$ \\ ${ }^{1}$ Astronomical Observatory, Volgina 7, 11160 Belgrade, Serbia \\ ${ }^{2}$ Astrophysikalisches Institut Potsdam, An der Sternwarte 16, 14482 Potsdam, Germany \\ ${ }^{3}$ Instituto de Astrofísica de Canarias C/ Vía Làctea, s/n E38200 - La Laguna, Tenerife, Spain \\ ${ }^{4}$ Department of Astronomy, Studentski trg 16, 11000 Belgrade, Serbia
}

\begin{abstract}
We present a study of the emission line profiles of a sample of 12 single-peaked Active Galactic Nuclei (AGNs- Sy 1 and QSOs). The narrow [OIII] lines seem to be related to two components; NLR1, which has dispersion velocities from 200 to $500 \mathrm{~km} \mathrm{~s}^{-1}$ and is blueshifted with respect to the systemic by about 20 to $350 \mathrm{~km} \mathrm{~s}^{-1}$; and NLR2, with smaller dispersion velocities $\left(\sim 100-200 \mathrm{~km} \mathrm{~s}^{-1}\right)$ and a redshift corresponding to the cosmological one. The broad lines have complex structure and a two-component model has been applied.
\end{abstract}

\section{Introduction}

The detection and modeling of some double-peaked Balmer lines has supported the idea about a disk geometry in the Broad Line Region - BLR (see e.g., Strateva et al. 2003; Storchi-Bergmann et al. 2003; Eracleous \& Halpern 2003 and references therein). On the one hand, the existence of double-peaked lines should not be required as a necessary condition for the existence of a disk geometry in BLRs. Even if the emission in a spectral line comes from a disk, the parameters of the disk (e.g. inclination) can be such that one observes single-peaked lines. On the other hand, taking into account the complexity of emission line regions of AGNs, one might expect that the broad emission lines are composed of radiation from two or more kinematically and physically different emission regions. Consequently, one of the possibilities could be that the emission of the disk is masked with emission of another emission line region.

Here we present a study of the emission line profiles of a sample of 12 single-peaked AGNs in order to investigate the structure of emission line region and to find evidence, which suggests that the disk emission can contribute to the line emission (even if they have single-peaked line profiles). To do this, we observed 12 AGNs in the $\mathrm{H} \alpha$ and $\mathrm{H} \beta$ wavelength region with the Isaac Newton Telescope, which provided the relatively high spectral resolution and $\mathrm{S} / \mathrm{N}$ ratio of the spectral lines.

\section{Observations and methods of analysis}

The observations were performed with the $2.5 \mathrm{~m}$ INT at La Palma in the period of 21 to 25 of January 2002. The Intermediate Dispersion Spectrograph (IDS) and the 235 camera (with chip EEV10) in combination with the R1200Y (for the $\mathrm{H} \alpha$ wavelength region) and $\mathrm{R} 1200 \mathrm{~B}$ (for the $\mathrm{H} \beta$ wavelength region) gratings was used. The seeing was around $1^{\prime \prime} .1$ and the slit width was $1^{\prime \prime}$. The spectral resolution was $\sim 1 \AA$.

To analyze the shape of the $\mathrm{H} \beta$ and $\mathrm{H} \alpha$ lines, we first use Gaussian decomposition (for more details see Popović et al. 2001, Popović et al. 2002, Popović et al. 2003). Second, we apply the two-component model in the modeling of broad line shapes of 12 AGNs, 
where one component is the disk or disk-like region and another one is from spherical emission. For the disk we use the Keplerian relativistic model of Chen et al. (1989). The kinematics of the additional emission region can be described as emission from a spherical region with isotropic velocity distribution. Consequently, the emission line profile of this emission region can be described by a Gaussian function. The whole line profile can be described by the relation: $I(\lambda)=I_{A D}(\lambda)+I_{G}(\lambda)$, where $I_{A D}(\lambda), I_{G}(\lambda)$ are the emission of the relativistic accretion disk and an added emission region, respectively.

Before performing the fitting we have 'cleaned' the spectra by subtracting narrow and satellite lines (see e.g. Popović et al. 2003) normalized the intensities of $\mathrm{H} \alpha$ and $\mathrm{H} \beta$ to one and convert the wavelength in velocity scale: $\lambda \rightarrow X=\left(\lambda-\lambda_{0}\right) / \lambda_{0}$. We found that in all AGNs of the sample the $\mathrm{H} \alpha$ and $\mathrm{H} \beta$ have similar profiles.

\section{Results}

Concerning the Gaussian analysis of broad components we can conclude: (i) that the $\mathrm{H} \beta$ and $\mathrm{H} \alpha$ line shapes of the AGNs considered are very complex, and usually cannot be described by one Gaussian. (ii) that the Gaussian decomposition indicates the existence of a central broad component with low random velocities (500 to $1500 \mathrm{~km} \mathrm{~s}^{-1}$ ) of a redshift consistent with the systemic velocity (between $\pm 500 \mathrm{~km} \mathrm{~s}^{-1}$ ). The red and blueshifted broad components are often present in the fit. These components tend to have higher random velocities with higher (positive or negative) shift. (iii) The widths of Fe II lines have different random velocities and redshift consistent with the systemic velocity $\left( \pm 500 \mathrm{~km} \mathrm{~s}^{-1}\right)$. Also, the Fe II random velocity tends to have a linear function with the central broad component random velocity, indicating that a significant fraction of Fe II emission is created in a region which contributes to the core of $\mathrm{H} \alpha$ and $\mathrm{H} \beta$.

The NLRs of the AGNs considered also show a complex structure, and we can clearly see at least two NLR regions in all observed AGNs: (i) the NLR1, which has an internal random velocity from $\sim 200$ to $500 \mathrm{~km} \mathrm{~s}^{-1}$, relative approaching velocities from 20 to $350 \mathrm{~km} \mathrm{~s}^{-1}$ with respect to the systemic redshift of the observed galaxy; and (ii) the NLR2 which has internal random velocity between $100-250 \mathrm{~km} \mathrm{~s}^{-1}$, and redshift which corresponds to systemic redshift of the corresponding object.

Here we apply the two-component model and the line profiles can be well fitted with this model. But without constraints in some of the parameters (e.g. emissivity index, inclination, inner and outer radii) it is not possible to find a unique solution for the parameters of the model. Unique solutions, require constraints is at least one of the disk parameters. But, it is obvious that in all AGNs from the sample, the shape of the line wings indicates strong radial motion, that may be caused by disk-like geometry. In any case, the fitting test allows us to give rough estimates of the kinematical parameters of the two-component model. See Popović et al. (2004) for a more detailed discussion.

\section{References}

Chen, K., Halpern, J. P., \& Filippenko, A. V. 1989, ApJ, 339, 742

Eracleous, M., \& Halpern, J. P. 2003, ApJ, 599, 886

Popović, L. Č. 2003, ApJ, 599, 140

Popović, L. Č., Mediavilla, E., Bon, E., \& Ilić D. 2004, A\&A, submitted

Popović, L. Č., Mediavlilla, E. G., Bon, E., Stanić, N., \& Kubičela, A. 2003, ApJ, 599, 185

Popović, L. Č., Mediavlilla, E. G., Kubičela, A., \& Jovanović, P. 2002, A\&A, 390, 473

Popović, L. Č., Stanić, N., Kubičela, A., \& Bon, E. 2001, A\&A, 367, 780

Storchi-Bergmann, T., et al. 2003, ApJ, 598, 956

Strateva, I. V., et al. 2003, AJ, 126, 1720 